\title{
Astrocyte-mediated short-term synaptic depression in the rat hippocampal CA1 area: two modes of decreasing release probability
}

\author{
My S Andersson ${ }^{*}$ and Eric Hanse
}

\begin{abstract}
Background: Synaptic burst activation feeds back as a short-term depression of release probability at hippocampal CA3-CA1 synapses. This short-term synaptic plasticity requires functional astrocytes and it affects both the recently active (< 1 s) synapses (post-burst depression) as well as inactive neighboring synapses (transient heterosynaptic depression). The aim of this study was to investigate and compare the components contributing to the depression of release probability in these two different scenarios.

Results: When tested using paired-pulses, following a period of inactivity, the transient heterosynaptic depression was expressed as a reduction in the response to only the first pulse, whereas the response to the second pulse was unaffected. This selective depression of only the first response in a high-frequency burst was shared by the homosynaptic post-burst depression, but it was partially counteracted by augmentation at these recently active synapses. In addition, the expression of the homosynaptic post-burst depression included an astrocyte-mediated reduction of the pool of release-ready primed vesicles.

Conclusions: Our results suggest that activated astrocytes depress the release probability via two different mechanisms; by depression of vesicular release probability only at inactive synapses and by imposing a delay in the recovery of the primed pool of vesicles following depletion. These mechanisms restrict the expression of the astrocyte-mediated depression to temporal windows that are typical for synaptic burst activity.
\end{abstract}

\section{Background}

The probability of release $\left(\mathrm{P}_{\mathrm{r}}\right)$ is a fundamental property of synapses that is regulated by presynaptic activity (shortterm synaptic plasticity) [1] and by modulatory transmitters acting on presynaptic receptors [2-4]. $\mathrm{P}_{\mathrm{r}}$ at rest (after seconds of inactivity) varies substantially among synapses [5] and is determined by two independent factors. One is the number of vesicles primed for release and thus potentially available for release by a single action potential, the primed pool. The other is the probability of releasing one primed vesicle $\left(\mathrm{P}_{\text {ves }}\right)[6,7]$. Repeated activation at short intervals, resulting in residual elevated calcium in the presynaptic terminal between activations, will change $P_{\text {ves }}$, rapidly deplete the primed pool, and prime new vesicles in a calcium-dependent manner [8]. During high-frequency activation $P_{r}$ is rather determined by the rate at which new

\footnotetext{
* Correspondence: my.andersson@physiol.gu.se

Institute of Neuroscience and Physiology, Gothenburg University, Göteborg, Sweden Box 432, Medicinaregatan 1140530 Göteborg, Sweden
}

vesicles can become available for release [7,9]. Thus, factors determining $P_{r}$ differ depending on whether the presynaptic terminal has been recently active, or not, and modulatory transmitters may modulate $\mathrm{P}_{\mathrm{r}}$ differently when synapses are active compared to following a period of rest [10].

We have found that activation of astrocytes by a short synaptic burst negatively modulates release probability at CA3-CA1 glutamate synapses [11]. From a period of hundreds of milliseconds to seconds after a short synaptic burst, $\mathrm{P}_{\mathrm{r}}$ is reduced in the recently active synapses (postburst depression, PBD). This PBD is absent when strongly buffering calcium in the astrocyte gap junctioncoupled network, when inhibiting astrocyte metabolism and early in development when the astrocyte network still not has gained its mature function. This short-term astrocyte-mediated depression is also observed as a reduction of $P_{r}$ in inactive neighboring synapses (transient heterosynaptic depression, tHeSD) [12]. Although $\mathrm{P}_{\mathrm{r}}$

\section{() Biomed Central}


is depressed in the PBD and in tHeSD it is unclear if these depressions are based on the same mechanism. One obvious difference between the PBD and the tHeSD is the recent presynaptic activity. In the present study we have therefore compared the PBD and the tHeSD with respect to estimated changes in $\mathrm{P}_{\mathrm{ves}}$ and primed pool.

\section{Results}

PBD and tHeSD are associated with different changes in the paired-pulse ratio

A relatively modest conditioning, a 3 -impulse $(50 \mathrm{~Hz})$ synaptic burst, in the hippocampal CA1 area, results in a substantial short-term homosynaptic (PBD) and heterosynaptic (tHeSD) transient astrocyte-mediated depression, respectively, half a second after the conditioning burst $[11,12]$. The experimental protocol for the PBD and the tHeSD is schematically shown in Figure 1A. Our standard protocol consisted of a 3-impulse, $50 \mathrm{~Hz}$, burst, 500 milliseconds before a paired-pulse test stimulus applied either homosynaptically (PBD), or heterosynaptically (tHeSD) every 10 seconds (Figure 1A). The control for the heterosynaptic depression was the paired-pulse test preceded $5 \mathrm{~s}$ before with a 3-impulse $(50 \mathrm{~Hz})$ synaptic burst, every protocol was repeated 18 times.

These two astrocyte-mediated depressions were both associated with increased paired-pulse ratio (PPR), but to different extent. We will use a change in PPR in association with the astrocyte-mediated depressions as an indication of a change in $\operatorname{Pr}$ [1] (see also Methods) since we previously have shown that that the PBD is also associated an equal depression reported by the AMPA and by the NMDA EPSCs, as well as by a matching decrease in the $1 / \mathrm{CV}^{2}[11]$. Here, we first wanted to, in more detail, examine the relationship between the depression and the increased PPR for the PBD and $t H e S D$, respectively. Under our experimental conditions (e.g. $4 \mathrm{mM}$ calcium and $4 \mathrm{mM}$ magnesium in the extracellular solution), the PPR was about 1.5, both when $20 \mathrm{~ms}$ or $50 \mathrm{~ms}$ was used as interstimulus interval (Figure 1B). The PPR increased to about 2 in association with the tHeSD, but only to about 1.6 in association with the PBD $(2.1 \pm 0.03, \mathrm{n}=29$ respectively $1.6 \pm 0.08 \mathrm{n}=29, \mathrm{p}<0.01)$, despite the fact that the PBD was expressed as a substantially larger depression of the field EPSP (fEPSP) than the tHeSD $(41.5 \pm 2 \%, \mathrm{n}=35$ respectively $20 \pm 2 \%, \mathrm{n}=29, \mathrm{p}<$ 0.001) (Figure 1B).

The large increase of the PPR associated with the tHeSD indicates that the second fEPSP in the test pairedpulse protocol should be little affected. Indeed, there was no change of the second fEPSP in association with the tHeSD (Figure 1C). Using a 3-impulse $50 \mathrm{~Hz}$ test stimulus (instead of the paired-pulse test) we found that also the third fEPSP of this 3-impulse test stimulus was not depressed $(101 \pm 6 \%, n=7, p>0.1)$. This result indicates that the depression of $\mathrm{P}_{\mathrm{r}}$ in association with the tHeSD is selective for stimuli that occur following a period of rest. In marked contrast to the tHeSD, the second fEPSP in the test paired-pulse protocol was significantly depressed in association with the PBD $(34 \pm 6 \%, \mathrm{n}=13$, $\mathrm{p}<0.01)$ (Figure 1D). The differential relationship between the amount of depression and the change of the PPR for the $\mathrm{PBD}$ and tHeSD, respectively, suggests that different mechanisms are involved in reducing $\mathrm{P}_{\mathrm{r}}$, raising the question how the PBD and tHeSD may interact.

\section{Occlusion between PBD and tHeSD}

To test for interaction between the PBD and the tHeSD we applied the 3-impulse burst simultaneously to the two synaptic inputs (Figure 2A) and compared the depression of the fEPSP $500 \mathrm{~ms}$ later to the PBD in the same experiment. As demonstrated in Figure 2A the addition of the 3 -impulse burst to the other synaptic input did not increase the amount of depression compared to the control PBD, indicating that the homosynaptic conditioning fully elicits the type of depression elicited by the heterosynaptic conditioning $(104.4 \pm 2 \%, \mathrm{n}=3, \mathrm{p}<0.1)$. Alternatively, recent synaptic activity renders synapses resistant to the type of depression elicited by the heterosynaptic conditioning. We next tested to elicit hetero- and homosynaptic depression sequentially by first applying the heterosynaptic conditioning and then, $500 \mathrm{~ms}$ later, the homosynaptic conditioning (Figure 2B). The heterosynaptic conditioning elicited a depression of $28 \pm 6 \%(n=8, p<0.001)$ of the fEPSP, associated with an increased PPR of $48 \pm 10 \%$ ( $\mathrm{n}=$ $8, \mathrm{p}<0.01$ ) (Figure 2B) (cf. Figure 1). The additional homosynaptic conditioning increased the depression to $38 \pm 3 \%,(\mathrm{n}=8, \mathrm{p}<0.05)$ but reduced the increase of the PPR to $25 \pm 8 \%(\mathrm{n}=8, \mathrm{p}<0.01)$ (Figure $2 \mathrm{C})$. These interaction experiments suggest that the homosynaptic PBD consist of two components reducing $\mathrm{P}_{\mathrm{r}}$, one in common with inactive synapses and one specific for recently active synapses. The depression component specific for the homosynaptic conditioning is associated with decreased PPR, possibly related to facilitation/augmentation induced concomitantly with depletion of primed vesicles by the homosynaptic conditioning.

To elucidate how the homosynaptic and heterosynaptic depressions interact we compared a multiplicative, and an additive relationship with the actual data. In these experiments the average actual PBD was $40 \pm 3 \%$ $(n=8)$. An additive relationship between the tHeSD and the remaining depression in the subsequent $\mathrm{PBD}$ predicts a total PBD of $48.5 \pm 4(\mathrm{n}=8)$. A multiplicative relationship predicts a total PBD of $43.8 \pm 3.1(n=8)$. Thus, these calculations suggest that a multiplicative interaction and an additive interaction both represent the data reasonable well, but they do not distinguish between the two models. 


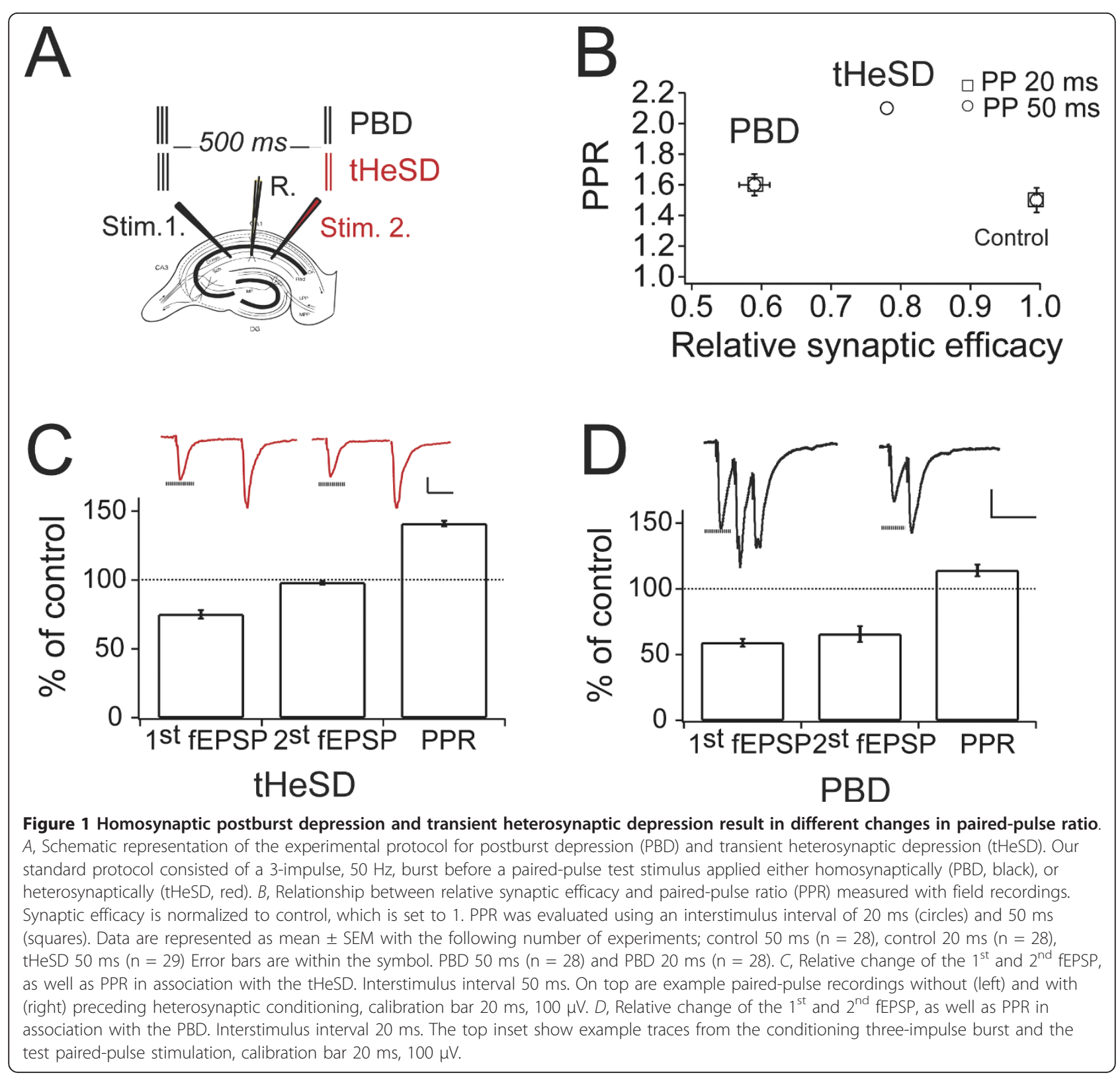

\section{PBD is associated with a decreased pool of primed vesicles}

In contrast to the tHeSD, which was associated with a selective depression of the first EPSP, also the second EPSP in the paired-pulse protocol was reduced in the PBD (albeit to a lesser extent than the first EPSP). To further examine the consequences of homosynaptic conditioning we used a train of 10 action potentials (at 50 $\mathrm{Hz}$ ) as a test stimulus, and used whole-cell voltageclamp recordings to monitor the responses (Figure 3A). Under these conditions the 3 -impulse conditioning burst caused a depression of the first EPSC in the train by $56 \pm 4 \%(\mathrm{n}=7, \mathrm{p}<0.001)$, which is somewhat larger than the depression observed using field recordings and a 2-impulse test stimulus (Figure 1). As shown in Figure $3 \mathrm{C}$, not only the first and second EPSC were depressed, but also the third and fourth, whereas the last six EPSCs in the test train were largely unaffected. Increasing the number of stimuli in the conditioning burst to 10 extended the depression of the test train to all synaptic responses (Figure 3B). A direct comparison of the depressions caused by 3 - and 10-impulse conditioning shows that, whereas the 10-impulse conditioning causes an overall larger depression, the depression of the first EPSC (54 $\pm 4 \%, n=7, p<0.001)$ is about the same in these two situations (Figure 3C) [11]. 


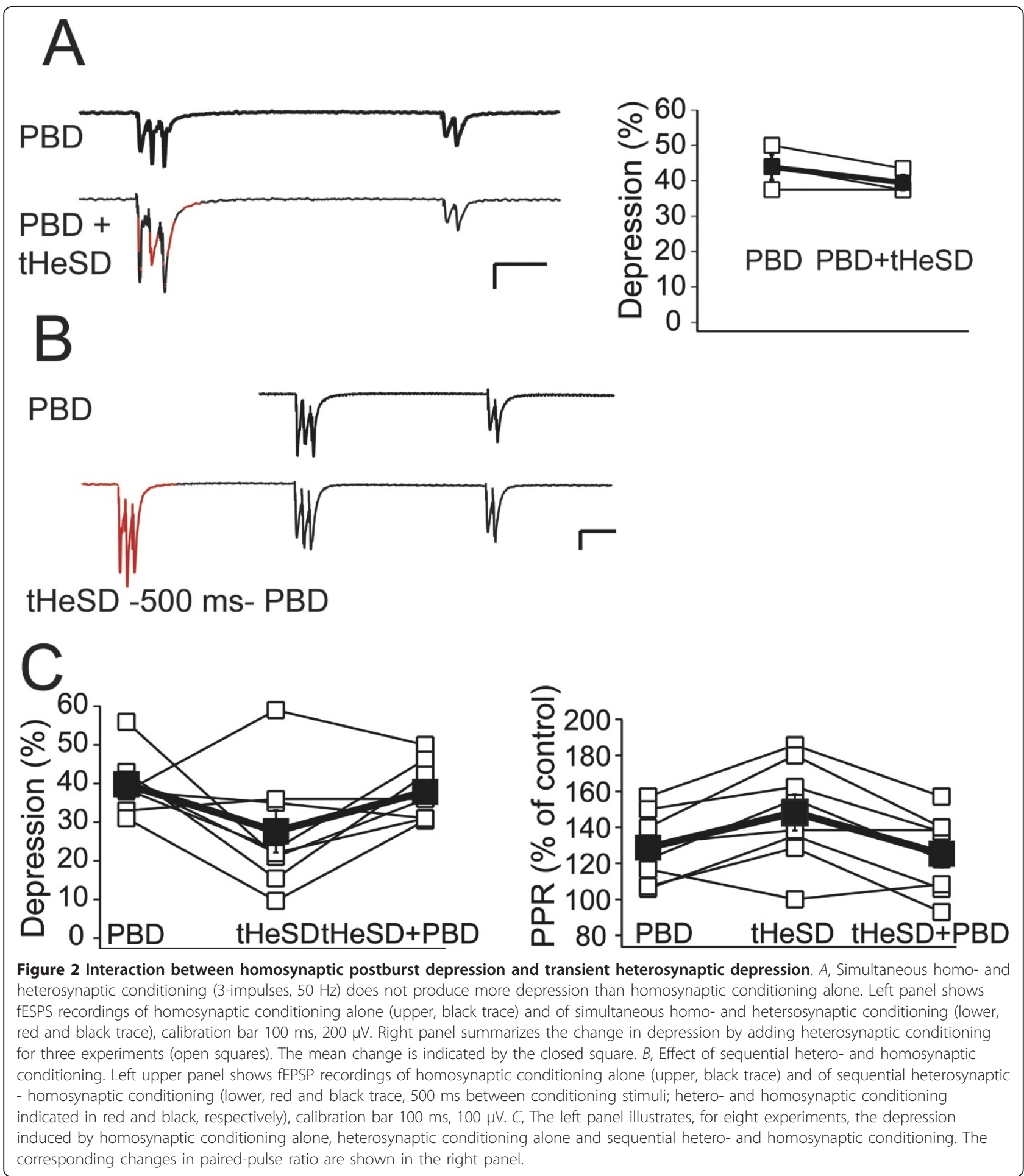

The overall response during train stimulation can be used to estimate the relative number of primed vesicles available for release at the onset of the train stimulation $[7,13,14]$. The rationale behind this estimation is that these vesicles are rapidly consumed by the first few stimuli in the train and priming of new vesicles starts rapidly in the presence of elevated intraterminal calcium. When pre-primed vesicles are consumed and the activity-dependent priming is fully developed, $\mathrm{P}_{\mathrm{r}}$ per stimulus becomes rather constant because the release is matched by the rate of new priming. By plotting the cumulative EPSC amplitude (expressed in units of the $1^{\text {st }}$ EPSC in 


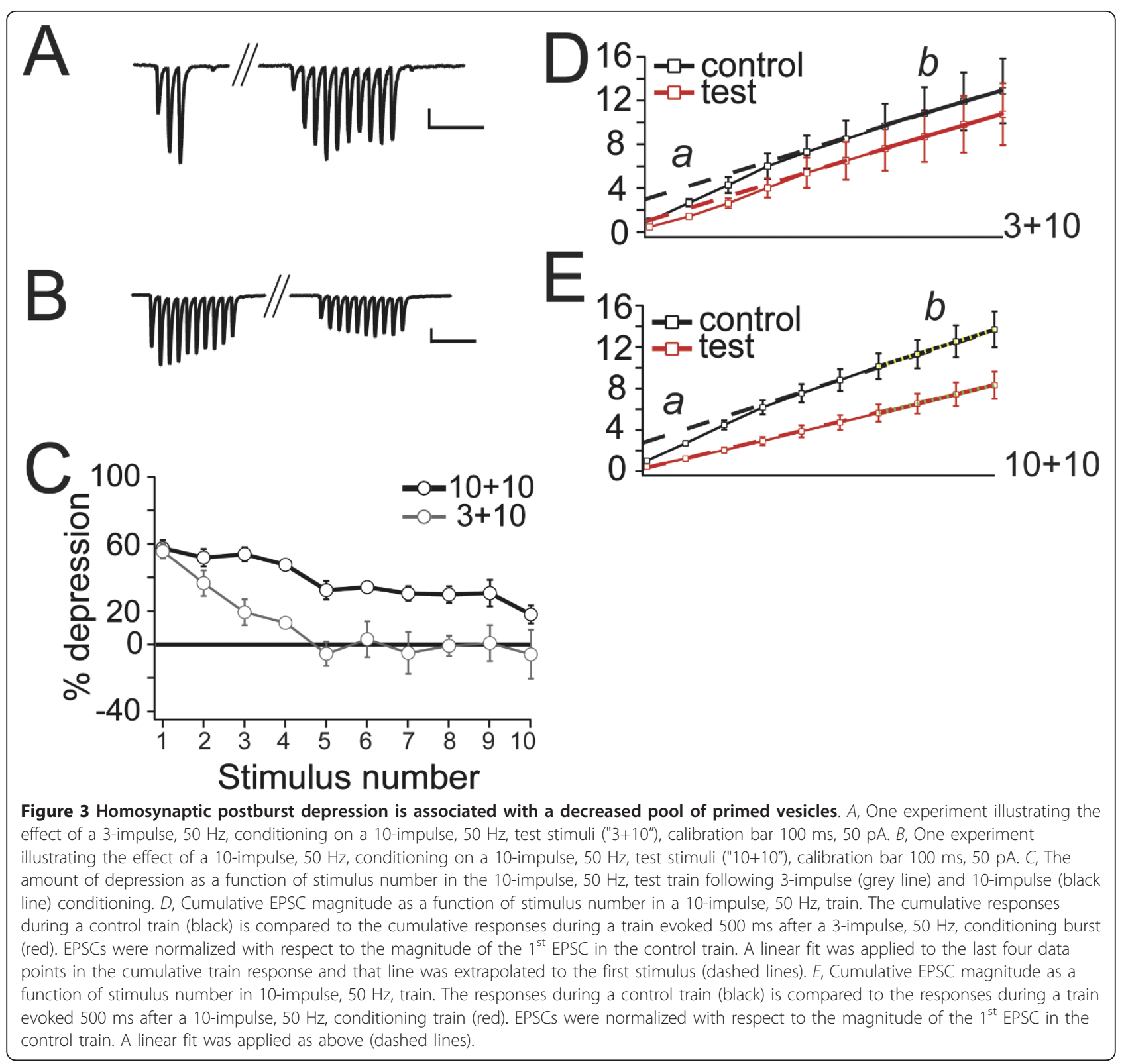

the control train) as a function of stimulus number (Figure $3 \mathrm{D}, \mathrm{E})$, the relative priming rate can be estimated as the slope of the linear late part of the cumulative EPSC stimulus number relationship. This analysis indicated that the activity-dependent priming rate was unaffected by the 3 -impulse conditioning $(1.1 \pm 0.04$ vs $1.1 \pm 0.03$, $\mathrm{n}=7$ ) (Figure $3 \mathrm{D})$, whereas it was reduced by the 10 impulse conditioning $(1.2 \pm 0.01$ vs $0.9 \pm 0.003, \mathrm{n}=7$, $\mathrm{p}<0.01$ ) (Figure 3E).

The number of (primed) vesicles available for release at the onset of the train stimulation can be estimated by subtracting the contribution from activity-dependent priming (recruitment) of new vesicles from the total response during the train. The relative magnitude of the estimate of the pre-primed pool will then depend on when during the train stimulation recruitment is assumed to start. Assuming full recruitment rate already at the first stimulus in the train, an estimate of the preprimed pool of vesicles can be obtained by the value of the extrapolated regression line at the first stimulus. With this representation of the pre-primed pool, we observed a reduction by $61 \pm 0.5 \%(n=, 7, \mathrm{p}<0.001)$ following the 3 -impulse conditioning and by $92 \pm 4 \%$ ( $n=$ $7, \mathrm{p}<0.001$ ) following the 10 -impulse conditioning. These values are most certainly an overestimate of the depression since the method of back extrapolating will underestimate the pre-primed pool size [cf. 13]. Nevertheless, this analysis indicates that the PBD is associated 
with a reduction of the pre-primed pool of vesicles and that this reduction increases when increasing the number of stimuli in the conditioning train.

A possible explanation for the similar magnitude of the PBD following a conditioning burst of 3 and 10 impulses (Figure 3C) is that the smaller pool (larger depletion) following the 10 impulse conditioning is compensated for by an increased $P_{\text {ves }}$, i.e. facilitation/augmentation [15], thereby maintaining $\mathrm{P}_{\mathrm{r}}[16]$. A burst consisting of 3-4 impulses, and of 10 impulses at $50 \mathrm{~Hz}$ have previously been shown to elicit an augmentation at these CA3-CA1 synapses (single exponential decay with a time constant of about eight seconds) of about $108 \%$ and $124 \%$ of control, respectively, two seconds after the burst [17]. If an increased facilitation/augmentation counteracts an increased depletion, one would expect that the PBD following the 10-impulse conditioning should be associated with a smaller PPR than the PBD following the 3-impulse conditioning, since facilitation/augmentation is associated with pronounced decrease in PPR [18]. Consistent with a larger depletion (associated with no change in PPR) and a larger facilitation/augmentation (associated with decreased PPR) the PPR was indeed smaller after the 10-impulse $(0.9 \pm 0.10, \mathrm{n}=7)$, than after the 3 impulse $(1.15 \pm 0.10, \mathrm{n}=7)$, conditioning $(\mathrm{p}<0.05)$.

The results so far indicate that PBD is a variable mixture of three different forms of short-term plasticity; the astrocyte-mediated depression of "resting $\mathrm{P}_{\text {ves }}$ ", facilitation/augmentation and depletion of primed vesicles. Since these three forms short-term plasticity are associated with qualitatively very different changes in PPR, an analysis of the changes in PPR might be an alternative approach to estimate the reduction in the size of the primed vesicle pool by the homosynaptic conditioning. Figure 4A illustrates the relationship between changes in synaptic efficacy and changes in PPR for PBDs and tHeSDs. Since these changes are expressed as ratios they are plotted on logarithmic scales. Changes in Pr solely based on a change in the pool of primed vesicles are expected to affect EPSP1 and EPSP2 about equally and thus not result in changes of the PPR [13,19], as indicated by the green line. Changes in Pr based solely on a change in "resting Pves" are, on the other hand, expected to only affect EPSP1 thus resulting in reciprocal changes of the PPR, as indicated by the blue line. As discussed above, the tHeSD (open square) is a depression of "resting Pves" and its coordinates also fall close to the green line. When astrocyte metabolism was inhibited by FAc [12] the same conditioning stimuli results in no depression and no change in PPR (open square with cross). The coordinates for facilitation (filled triangle), and likely augmentation [18], are also close to the green line, indicating that astrocyte-mediated depression of "resting Pves" and facilitation/augmentation cause reciprocal changes of synaptic efficacy and PPR. The coordinates for PBD elicited by a 3-impulse burst (filled circle), by a 10-impulse burst (empty circle) and by a 3-impulse burst when astrocyte signaling was inhibited (open circle with cross, [11]) by either intracellular calcium chelation, or metabolic inhibition, are all relatively close to the green line.

To estimate how much a reduction in the pool contributes to the PBD elicited by a 3-impulse burst (Figure 4B), we assume that the three different short-term plasticities ("resting $\mathrm{P}_{\text {ves }}$ " depression, facilitation/augmentation and depletion) multiply up to a net PBD. If "resting $\mathrm{P}_{\text {ves }}$ " depression and facilitation/augmentation only occur in separate synapses (see also Discussion) one should instead have assumed an additive relationship. However, the quantitative difference between a multiplicative and additive relationship is not large using relevant magnitudes of these plasticities. For example, if "resting $\mathrm{P}_{\text {ves }}$ " reduces synaptic efficacy to 0.75 and if facilitation/augmentation increases synaptic efficacy to 1.33 their combined action should be 1.0 using the multiplicative relationship and 1.08 using the additive relationship. The relationship between $P_{\text {ves }}$ and vesicle pool is likely sub-multiplicative [6,7], but for rather small absolute values of $P_{\text {ves }}$ and vesicle pool, and rather small changes in these parameters, a multiplicative relationship is a reasonable approximation. For example, if Pves is 0.2 and the number of primed, release ready, vesicles is 2 , a reduction by a factor 2 of both these parameters will reduce $\mathrm{P}_{\mathrm{r}}$ from 0.36 to 0.1 (72\%) using the sub-multiplicative relationship and from 0.4 to 0.1 (75\%) using the multiplicative relationship, an error by about $4 \%$.

Based on the increase of PPR in association with the tHeSD $(1.41 \pm 0.0024, n=28)$ of control, Figure 1$)$ and the full occlusion between the tHeSD and the PBD (Figure 2), we first estimate that the "resting $\mathrm{P}_{\text {ves }}$ " depression contributes with 0.71 of control $(1 / 1.41)$ to the PBD elicited by a 3 -impulse burst (Figure 4B, blue arrow). Since PPR associated with the PBD was $1.15 \pm 0.05, \mathrm{n}=28$ of control and "resting $\mathrm{P}_{\text {ves }}$ " depression should contribute by 1.41 , facilitation/augmentation should contribute with a reduction in PPR by 0.82 (1.15/1.41), and an facilitation/augmentation associated with a PPR of 0.82 of control should correspond to an increase of the first EPSP (EPSC) by $1.22(1 / 0.82$, Figure $4 \mathrm{~B}$ grey arrow). Having estimated the contribution from "resting $\mathrm{P}_{\text {ves }}$ " depression (0.71) and from facilitation/ augmentation (1.22) we estimated the contribution from depletion as the total PBD $(0.59, \pm 0.03, \mathrm{n}=28)$ divided by the product 0.71 and 1.22 , yielding a value of 0.68 of control (32\% depression, Figure 4B green arrow).

Using the same calculation we found that the estimated contribution of depletion to the PBD following the 10-impulse burst (Figure 4C) had increased to 0.54 (46\% depression). With this method based on relative changes in PPR to estimate the depletion we thus find 


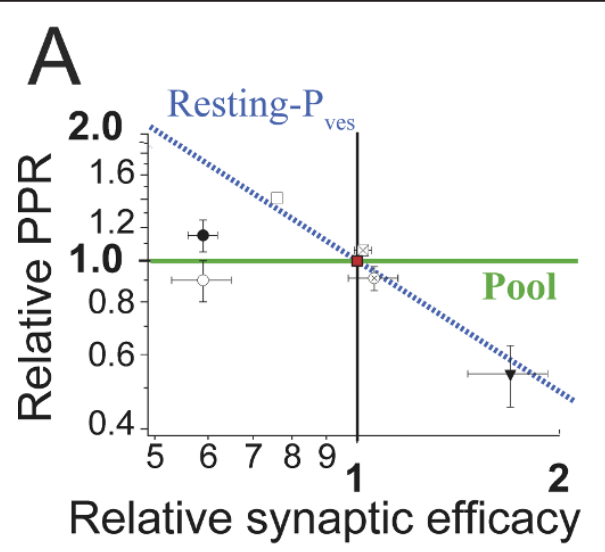

\section{B}
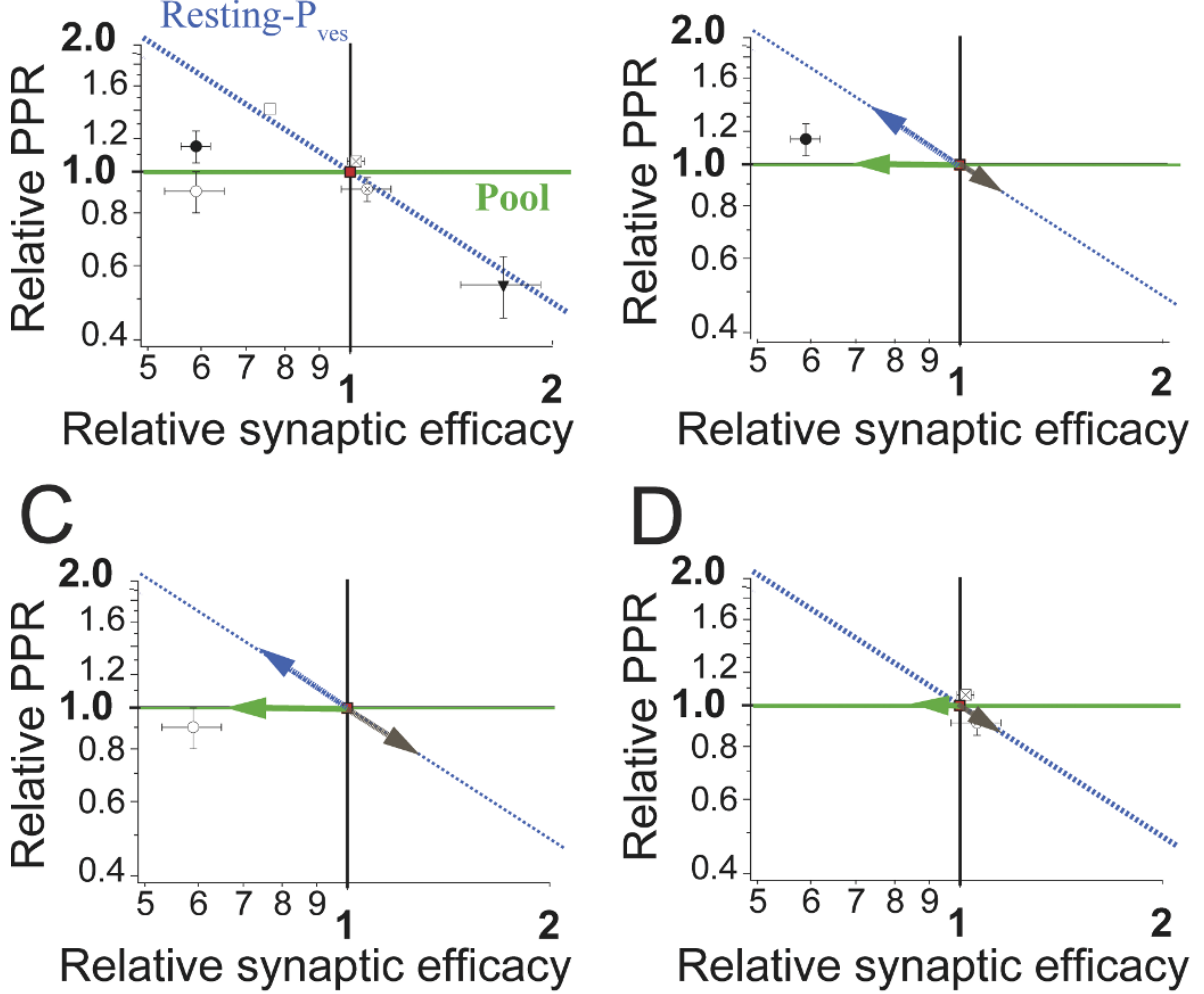

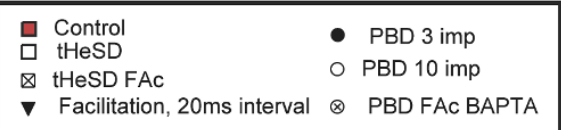

Figure 4 Estimation of facilitation/augmentation and depletion following homosynaptic conditioning using the paired-pulse ratio A. Relationship between relative synaptic weight and relative PPR for different conditioning (indicated in the figure with different symbols) normalized with respect to control. Control synaptic weight and control PPR are set to 1. Dotted blue line indicates the expected relationship

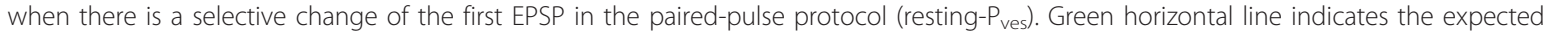
relationship when there are equal changes of the first and of the second EPSP (pool). Error bars for the theSD are within the symbol. $B$. Estimation of the contribution of "resting Pves" depression (blue arrow), facilitation/augmentation (grey arrow) and depletion (blue arrow) to the postburst depression $500 \mathrm{~ms}$ after a 3-impulse $(50 \mathrm{~Hz}$ ) burst. C. Same as B, but for the postburst depression $500 \mathrm{~ms}$ after a 10 -impulse (50 Hz) burst. D. Same as B, but for the postburst depression $500 \mathrm{~ms}$ after a 3-impulse $(50 \mathrm{~Hz})$ burst when astrocyte signaling was compromised either by 50 mM BAPTA intracellularly, or $1 \mathrm{mM}$ fluoroacetate extracellularly (Andersson and Hanse, 2010).

that depletion is $44 \%$ larger following the 10 -impulse burst compared to that following the 3-impulse burst. This increased depletion is somewhat smaller, but comparable, to the increase we found using the method of cumulative burst response (51\%, cf. Figure 3).

Compromising astrocyte signaling using either intracellular calcium chelation, or inhibition of the citric acid cycle resulted in a blockade of the PBD [11]. In fact, a small potentiation of $1.06( \pm 0.09, \mathrm{n}=9)$ of control associated with a decrease of the PPR to $0.91( \pm 0.06, n=9)$ of control remained (Figure 4D). Since the "resting $\mathrm{P}_{\text {ves" }}$ " depression is expected to be to totally blocked under these conditions (Figure 4A) facilitation/augmentation alone should account for the change in PPR, that is, 1.10 (1/0.91). The estimated depletion under these conditions should then be 0.94 ( $6 \%$ depression), that is, substantially smaller than when astrocyte signaling was intact (32\%, Figure 4B).

\section{Discussion}

The present study has examined how astrocyte-mediated short-term depression affects release probability at glutamate synapses in the CA1 hippocampal region. Our main conclusions are that activation of astrocytes decreases vesicular release probability at inactive synapses ("resting $\mathrm{P}_{\text {ves }}$ "), but not at active synapses 
("active $\mathrm{P}_{\text {ves }}$ "), and impose a delay in the recovery of primed vesicles following depletion by high-frequency activity.

\section{Depression of "resting $\mathbf{P}_{\text {ves", }}$ ", but not "active $\mathbf{P}_{\text {ves" }}$}

The tHeSD was associated with a selective depression of the first EPSP in a paired-pulse, or a high-frequency burst, protocol. The second and third EPSP evoked at $50 \mathrm{~Hz}$ were not depressed, but, if anything, slightly increased (Figure 1). This finding cannot be explained by a reduction in the number of release-ready (primed) vesicles, or by a general reduction in $\mathrm{P}_{\mathrm{ves}}$, both of which are not expected to be restricted to only the first EPSP. It is also not consistent with reduced calcium influx which affects both EPSPs (although not uniformly) evoked by a paired-pulse stimulus [e.g. 20]. To explain the selective depression of the first EPSP we propose that "resting" and "active" $\mathrm{P}_{\text {ves }}$ are differentially modulated such that astrocyte-mediated depression selectively affects "resting $\mathrm{P}_{\text {ves }}$ ". This proposal is in line with the finding that, although there is a large heterogeneity among developing hippocampal glutamate synapses regarding "resting $\mathrm{P}_{\text {ves }}$ ", "active $\mathrm{P}_{\text {ves }}$ " is rather uniform among these synapses [9].

Although we are not aware of any previous description of $\mathrm{P}_{\mathrm{r}}$ modulation restricted to "resting $\mathrm{P}_{\mathrm{ves}}$ ", this behavior is strikingly similar to the change in synaptic transmission produced by genetic elimination of the Rab3A-D [20]. Hippocampal synapses from these mice showed about $30 \%$ reduction in $\mathrm{P}_{\mathrm{r}}$ when tested with low frequency, but little, or no, reduction of $\mathrm{P}_{\mathrm{r}}$ when tested at high frequency. These findings from the Rab3-deficient mice indicated that Rab3s are involved in "superpriming" of vesicles in a subset of synapses [20]. Synapses with "superprimed" vesicles may well correspond to the subpopulation of high- $\mathrm{P}_{\mathrm{r}}$ synapses among the CA3-CA1 synapses [6,21-23]. Thus, a possible explanation for the tHeSD would be an astrocyte-mediated reversal of Rab3dependent "superpriming" at high-Pr synapses. According to the time-course of tHeSD $[12,24]$ this putative reversal of "superpriming" would develop during a few hundred ms and vanish within a few seconds.

\section{A combination of depression of "resting Pves", depletion of vesicles and augmentation during the PBD}

The interaction between hetero- and homosynaptic conditioning indicated that the homosynaptic PBD consists of two separable components (Figure 2). One component is shared with the tHeSD and should then be expressed as a depression of "resting $\mathrm{P}_{\mathrm{ves}}$ " and an increased PPR, possibly related to reversal of Rab3-dependent "superpriming" at high- $\mathrm{P}_{\mathrm{r}}$ synapses. The other component is then specific for recently active synapses and seems to involve depletion of vesicles, contributing to further depression, and facilitation/augmentation, counteracting the depression.

That the conditioning burst causes depletion of vesicles available for release by the subsequent test stimulation was indicated by the analysis of the cumulative test train response (Figure 3). Another analysis, based on changes in PPR (Figure 4), supported this conclusion. This analysis was based on the premises that facilitation/augmentation is associated with decreased PPR [18] whereas depletion is associated with no, or very small, changes of PPR [13,19].

The presence of facilitation/augmentation was indicated by reduced PPR (and more so with longer conditioning trains) associated with the component of the PBD specific for the homosynaptic conditioning (Figure 4). In addition to the potentiation per se, facilitation/augmentation may contribute to counteract the depression (of the first EPSP) and to the decreased PPR in another way. Since facilitation/augmentation implies a shift from "resting $\mathrm{P}_{\mathrm{ves}}$ " to "active $\mathrm{P}_{\text {ves }}$ ", it will render synapses expressing it resistant to the astrocyte-mediated depression of "resting $\mathrm{P}_{\mathrm{ves}}$ ". This scenario presupposes that facilitation/augmentation and the astrocyte-mediated depression of "resting $\mathrm{P}_{\text {ves }}$ " can be expressed in the same synapse. As will be outlined below, this is, however, not likely the case. Augmentation is typically expressed at low- $\mathrm{P}_{\mathrm{r}}$ synapses and appears to rather specifically potentiate only the first EPSC in a high-frequency train, i.e. "resting $\mathrm{P}_{\text {ves }}$ ", [18] and is thus associated with a prominent decrease of the PPR $[18,25]$. The magnitude of augmentation increases with increased number of stimuli in the conditioning high-frequency stimulation, but its decay time constant is characteristically remarkably invariable, being 5-10 s [1]. Although it remains to be determined with more accuracy, the decay time constant of the astrocyte-mediated depression of "resting $\mathrm{P}_{\mathrm{ves}}$ " is in the order of about $1 \mathrm{~s}[12,24]$, possibly explaining the finding that facilitation/augmentation is larger $2 \mathrm{~s}$, compared to $1 \mathrm{~s}$, following a conditioning burst [17]. This finding is not consistent with astrocyte-mediated depression of "resting $\mathrm{P}_{\mathrm{ves}}$ " and augmentation occurring in the same synapses since, if they were, facilitation/augmentation would have precluded the expression of the "resting $\mathrm{P}_{\text {ves" }}$ " depression. In support for the idea that these two shortterm synaptic plasticities occur in separate synapses our results indicated that, whereas facilitation/augmentation increased when increasing the number of stimuli in the conditioning train from 3 to 10, the depression of "resting $\mathrm{P}_{\text {ves }}$ " did not increase, but remained at the same magnitude. As discussed above it is likely that augmentation occurs at low- $\mathrm{P}_{\mathrm{r}}$ synapses while astrocyte-mediated depression of "resting $\mathrm{P}_{\text {ves }}$ " occurs at high- $\mathrm{P}_{\mathrm{r}}$ synapses.

We thus favor a scenario in which the conditioning burst elicits three different forms of short-term synaptic plasticity; a depression of "resting $\mathrm{P}_{\text {ves }}$ " (possibly at high- $\mathrm{P}_{\mathrm{r}}$ 
synapses), facilitation/augmentation of "resting $\mathrm{P}_{\text {ves }}$ " (possibly at low- $\mathrm{P}_{\mathrm{r}}$ synapses) and depletion of primed vesicles at all synapses. The relative contribution of these different forms of short-term plasticity will depend on the nature of the conditioning stimuli, on the conditioning - test interval, as well as on the relative number of low $\mathrm{P}_{\mathrm{r}}$ synapses in the synapse population.

\section{Astrocyte signaling imposes a delay in the recovery of primed vesicles}

We have previously shown that inhibiting astrocyte metabolism and calcium signaling prevents the tHeSD and the PBD $[11,12]$. When analyzed in some more detail here (Figure 4), the prevention of the PBD was associated with decreased PPR, indicating the presence of facilitation/ augmentation sufficient to just oppose depletion. Further analysis indicated that the depletion component was substantially reduced compared to the control situation, when astrocyte signaling was intact. The inhibition of astrocyte signaling did not affect the magnitude conditioning burst response, indicating that the size of preprimed pool of vesicles as well as the depletion of vesicles per se by the conditioning burst is unaffected by astrocyte signaling. Therefore, to explain the larger pool $0.5 \mathrm{~s}$ after a conditioning burst we propose that recovery from depletion is faster when astrocyte signaling is inhibited, or conversely, that an astrocyte signal impose a delay of the re-priming following depletion. This proposal has a precedent from results at the Calyx of Held synapse at which activation of metabotropic glutamate autoreceptors slowed down the recovery after the burst, while not affecting the response during the continuous high-frequency activation [26]. To what extent activation of metabotropic glutamate receptors is involved in the astrocyte-mediated delay of re-priming after a conditioning burst will be examined in future studies.

Recovery of primed vesicles is thought to be mediated by a fast calcium-dependent (few hundred ms) and a slow calcium-independent (2-6 s) mechanism [8]. Increased presynaptic calcium concentration accelerates recovery of primed vesicles [27-31]. This mechanism relies on calmodulin [29] binding to Munc-13 [28], and is counteracted by presynaptic $\mathrm{GABA}_{\mathrm{B}}$ receptor activation reducing presynaptic cAMP levels [10]. Since the recruitment rate during a 10-impulse, $50 \mathrm{~Hz}$, train following a 3-impulse burst was unaffected (Figure 3 ) it is unlikely that the presently proposed astrocyte-mediated delay of vesicle recovery acts by directly inhibiting the calcium-dependent priming. Therefore we propose that astrocyte signaling rather acts by slowing down calcium-independent recruitment, or by accelerating the decay of the calcium-dependent recruitment when stimulation stops (which is normally very rapid, in the order of $0.1 \mathrm{~s}$ [8]). As calcium-dependent recruitment can be modulated by the levels of cAMP
$[10,28]$ a putative glio-transmitter could mediate an acceleration of fast recruitment-decay by binding to a metabotropic receptor and decrease cAMP [32,33].

\section{Functional considerations}

Our results suggest that a short burst activation of astrocytes results in a transient depression of "resting $\mathrm{P}_{\mathrm{ves}}$ " and a delay in the replenishment of primed vesicles after depletion. We propose that other mechanisms controlling $\mathrm{P}_{\mathrm{r}}$, such as "active $\mathrm{P}_{\text {ves }}$ ", calcium-dependent recruitment and the size of release-ready primed vesicles, are not affected by the astrocyte signaling. These considerations may help to explain during which type of synaptic activity astrocytemediated short-term synaptic depression is operating. Thus, conditions during which there is residual calcium in the terminal, resulting in "active $\mathrm{P}_{\mathrm{ves}}$ " and calcium-dependent recruitment of new vesicles, are expected to preclude astrocyte-mediated depression. In line with this we found that continuous high-frequency trains were unaffected by inhibition of astrocyte signaling. We also found that continuous low-frequency trains were unaffected by inhibition of astrocyte signaling [11]. This finding is likely rather explained by insufficient temporal summation to activate the astrocytes. On the other hand, astrocyte-mediated short-term synaptic depression is expected to be prominently expressed during burst activity resembling theta burst activity (interburst frequencies of $2-10 \mathrm{~Hz}$, intraburst frequencies $>50 \mathrm{~Hz}$, and $>3$ impulses in each burst) [34].

\section{Conclusion}

Our results suggest that activated astrocytes depress the release probability via two different mechanisms; by depression of vesicular release probability only at inactive synapses and by imposing a delay in the recovery of the primed pool of vesicles following depletion. These mechanisms restrict the expression of the astrocytemediated depression to temporal windows that are typical for synaptic burst activity.

\section{Methods}

\section{Slice preparation and solutions}

Experiments were performed on hippocampal slices from 20- 50 day-old Wistar rats. The animals were killed in accordance with the guidelines of the local ethical committee for animal research (ref. 2008-2010-210). Rats were anaesthetized with isoflurane (Abbott) prior to decapitation. The brain was removed and placed in an ice-cold solution containing (in $\mathrm{mM}$ ): 140 cholineCl, $2.5 \mathrm{KCl}, 0.5$ $\mathrm{CaCl}_{2}, 7 \mathrm{MgCl}_{2}, 25 \mathrm{NaHCO}_{3}, 1.25 \mathrm{NaH}_{2} \mathrm{PO}_{4}, 1.3$ ascorbic acid and 7 dextrose. Transverse hippocampal slices (300 $400 \mu \mathrm{m}$ thick) were cut with a vibratome (HM 650V Microm, Germany) in the same ice-cold solution. Slices were subsequently stored in artificial cerebrospinal fluid (ACSF) containing (in $\mathrm{mM}$ ): $124 \mathrm{NaCl}, 3 \mathrm{KCl}, 2 \mathrm{CaCl}_{2}$, 
$4 \mathrm{MgCl}_{2}, 26 \mathrm{NaHCO}_{3}, 1.25 \mathrm{NaH}_{2} \mathrm{PO}_{4}, 0.5$ ascorbic acid, 3 myo-inositol, 4 D, L-lactic acid, and 10 D-glucose. After at least one hour of storage at $25^{\circ} \mathrm{C}$, a single slice was transferred to a recording chamber where it was kept submerged in a constant flow $\left(\sim 2 \mathrm{ml} \mathrm{min}^{-1}\right)$ at $30-32^{\circ} \mathrm{C}$. The perfusion fluid contained (in $\mathrm{mM}$ ) $124 \mathrm{NaCl}, 3 \mathrm{KCl}$, $4 \mathrm{CaCl}_{2}, 4 \mathrm{MgCl}_{2}, 26 \mathrm{NaHCO}_{3}, 1.25 \mathrm{NaH}_{2} \mathrm{PO}_{4}$, and $10 \mathrm{D}$ glucose. Picrotoxin $(100 \mu \mathrm{M})$ was and D-AP5 $(50 \mu \mathrm{M})$ was present in the perfusion fluid to block $\mathrm{GABA}_{\mathrm{A}}$ and NMDA receptor-mediated activity, respectively. All solutions were continuously bubbled with $95 \% \mathrm{O}_{2}$ and $5 \%$ $\mathrm{CO}_{2}$ ( $\mathrm{pH} \sim 7.4$ ). The higher concentration of $\mathrm{Ca}^{2+}$ and $\mathrm{Mg}^{2+}$ than normal was used to inhibit network activity. In a subset of experiments a cut was made between area CA3 and area CA1. However, since we observed the same amount depression in slices with and without a cut, the data from these two sets of experiments were pooled.

\section{Field recordings and analysis}

Electrical stimulation of Schaffer collateral/commissural axons and recordings of synaptic responses were carried out in the stratum radiatum of the CA1 region. Stimuli consisted of biphasic constant current pulses $(15-80 \mu \mathrm{A}$, 200 HS, STG 1002 Multi-Channel Systems MCS Gmbh, Reutlingen, Germany) delivered through tungsten wires (resistance $\sim 0.1 \mathrm{M} \Omega$,). One stimulation electrode was positioned in the stratum radiatum with a distance of $100 \mu \mathrm{m}$ from the registration electrode (Figure 1A). The synaptic input was activated every $10 \mathrm{~s}$ and stimulation intensity was adjusted so that spike activity was observed on the second or third fEPSP (but not on the first fEPSP) in the conditioning train. Field EPSPs were recorded with a glass micropipette (filled with perfusion fluid or $1 \mathrm{M}$ $\mathrm{NaCl}$, resistance 1-2 M 2 ). Field EPSPs were sampled at $10 \mathrm{kHz}$ with an EPC-9 amplifier (HEKA Elektronik, Lambrecht, Germany) and filtered at $1 \mathrm{kHz}$. Evoked responses were analyzed off-line using custom-made IGOR Pro (WaveMetrics, Lake Oswego, OR) software. Field EPSP magnitude was estimated by linear regression over the first $0.8 \mathrm{~ms}$ of the initial slope. Paired-pulse ratio (PPR) was calculated as the ratio of the initial slope of the EPSP2 (EPSC2) divided by the initial slope of the EPSP1 (EPSC1). The initial slope was measured using linear regression of only the first $0.8 \mathrm{~ms}$ of the EPSP (EPSC) initial slope to avoid possible influence by spike activity. $\mathrm{GABA}_{\mathrm{A}}$ receptors were blocked by picrotoxin precluding influence of $\mathrm{GABA}_{\mathrm{A}}$ receptor mediated IPSPs (IPSCs) on the PPR. Although a change in PPR is widely used as an indicator of a change in $\operatorname{Pr}[1,19]$, PPR can change without a change in Pr. For example, changes in synaptic efficacy (including silencing/unsilencing) caused by mechanisms other than a change in Pr, but only in a subpopulation of synapses whose mean Pr differs from the mean Pr of the synapse population recorded from, will change the PPR [cf. 35]. Moreover, postsynaptic mechanisms including AMPA receptor saturation, desensitization or polyamine unblock of calcium permeable AMPA receptors may contribute to changes in PPR, but such contributions are thought be negligible at CA3-CA1 synapses using interstimulus intervals of $20 \mathrm{~ms}$, or longer [36-41]. The presynaptic volley was measured as the slope of the initial positive-negative deflection, and it was not allowed to change by more than $10 \%$ during the experiment.

\section{Patch clamp recordings pyramidal cells and astrocytes}

Pyramidal cells were visually identified in the CA1 area with IR-DIC video microscopy (Nikon) and patched with an intracellular solution containing (in mM): $120 \mathrm{Cs}-$ methane sulphonate, $2 \mathrm{NaCl}, 10$ HEPES, 5 Qx-314, 4 MgATP, 0.4 GTP and 20 BAPTA ( $\mathrm{pH} \sim 7,2$ and osmolality to 295-300 mosmol). For measuring AMPA responses the cell was held at $-80 \mathrm{mV}$. Evoked responses were analyzed off-line using IGOR Pro (WaveMetrics, Lake Oswego, OR) software. Amplitudes were measured on average-sweeps of 18 consecutive sweeps. Astrocytes in the stratum radiatum were identified by their small soma and, when patched, for their linear responses to voltage steps [42]. The intracellular solution contained (in $\mathrm{mM}$ ): $120 \mathrm{KCl}, 2$ $\mathrm{NaCl}, 20$ HEPES, 4 Mg-ATP, 0.4 GTP and 50 BAPTA. Patch pipettes $(1.5 \mathrm{~mm} / 0.86 \mathrm{~mm}$; borosilicate, Clark Electrochemical Instruments) were pulled with a horizontal puller (Sutter Instruments Inc.) to a resistance of 3-6 $\mathrm{M} \Omega$. Series resistance was measured using a $5 \mathrm{~ms}, 10 \mathrm{mV}$ hyperpolarising pulse and was not allowed to change more than $15 \%$ during the experiment.

Data are expressed as means \pm SEM. Statistical significance for paired and independent samples was evaluated using Student's t - test.

\section{Drugs}

Drugs were from Tocris Cookson (Bristol, UK) except picrotoxin and fluoroacetate from Sigma-Aldrich (Stockholm, Sweden) and D-AP5 from Accent Scientific.

\section{Acknowledgements}

This work was supported by the Swedish Research Council (project number 12600), the Sahlgrenska Hospital (Agreement concerning Research and Education of Doctors), Wilhelm and Martina Lundgrens foundation, the Swedish Alzheimer's foundation, The Lars Hierta Memorial Foundation and The Swedish Society of Medicine.

\section{Authors' contributions}

All authors read and approved the final manuscript. MA participated in experiment design, performed the experiments, analyzed the data and prepared the manuscript. EH conceived of the study, and participated in its design and preparation of the manuscript.

Received: 4 April 2011 Accepted: 24 August 2011

Published: 24 August 2011 


\section{References}

1. Zucker RS, Regehr WG: Short-term synaptic plasticity. Annu Rev Physiol 2002, 64:355-405.

2. Pinheiro PS, Mulle C: Presynaptic glutamate receptors: physiological functions and mechanisms of action. Nat Rev Neurosci 2008, 9(6):423-436.

3. MacDermott AB, Role LW, Siegelbaum SA: Presynaptic ionotropic receptors and the control of transmitter release. Annu Rev Neurosci 1999, 22:443-485.

4. Miller RJ: Presynaptic receptors. Annual review of pharmacology and toxicology 1998, 38:201-227.

5. Branco T, Staras K: The probability of neurotransmitter release: variability and feedback control at single synapses. Nat Rev Neurosci 2009, 10(5):373-383.

6. Hanse E, Gustafsson B: Vesicle release probability and pre-primed pool at glutamatergic synapses in area CA1 of the rat neonatal hippocampus. J Physiol 2001, 531(Pt 2):481-493.

7. Sakaba T, Schneggenburger R, Neher E: Estimation of quantal parameters at the calyx of Held synapse. Neurosci Res 2002, 44(4):343-356.

8. Neher E, Sakaba T: Multiple roles of calcium ions in the regulation of neurotransmitter release. Neuron 2008, 59(6):861-872

9. Hanse E, Gustafsson B: Factors explaining heterogeneity in short-term synaptic dynamics of hippocampal glutamatergic synapses in the neonatal rat. J Physio/ 2001, 537(Pt 1):141-149.

10. Sakaba T, Neher E: Direct modulation of synaptic vesicle priming by $\mathrm{GABA}(\mathrm{B})$ receptor activation at a glutamatergic synapse. Nature 2003 , 424(6950):775-778

11. Andersson M, Hanse E: Astrocytes impose postburst depression of release probability at hippocampal glutamate synapses. J Neurosci 2010, 30(16):5776-5780.

12. Andersson M, Blomstrand $F$, Hanse E: Astrocytes play a critical role in transient heterosynaptic depression in the rat hippocampal CA1 region. J Physiol 2007, 585(Pt 3):843-852.

13. Abrahamsson T, Gustafsson B, Hanse E: Synaptic fatigue at the naive perforant path-dentate granule cell synapse in the rat. J Physiol 2005, 569(Pt 3):737-750.

14. Schneggenburger $\mathrm{R}$, Meyer AC, Neher E: Released fraction and total size of a pool of immediately available transmitter quanta at a calyx synapse. Neuron 1999, 23(2):399-409.

15. Stevens CF, Wesseling JF: Augmentation is a potentiation of the exocytotic process. Neuron 1999, 22(1):139-146.

16. Garcia-Perez E, Wesseling JF: Augmentation controls the fast rebound from depression at excitatory hippocampal synapses. J Neurophysiol 2008, 99(4):1770-1786

17. Gustafsson B, Asztely F, Hanse E, Wigstrom H: Onset Characteristics of Long-Term Potentiation in the Guinea-Pig Hippocampal CA1 Region in Vitro. Eur J Neurosci 1989, 1(4):382-394.

18. Granseth B, Lindstrom S: Augmentation of corticogeniculate EPSCs in principal cells of the dorsal lateral geniculate nucleus of the rat investigated in vitro. J Physiol 2004, 556(Pt 1):147-157.

19. Hanse E, Gustafsson B: Paired-pulse plasticity at the single release site level: an experimental and computational study. J Neurosci 2001, 21(21):8362-8369.

20. Schluter OM, Basu J, Sudhof TC, Rosenmund C: Rab3 superprimes synaptic vesicles for release: implications for short-term synaptic plasticity. J Neurosci 2006, 26(4):1239-1246.

21. Dobrunz LE, Stevens CF: Heterogeneity of release probability, facilitation and depletion at central synapses. Neuron 1997, 18(6):995-1008.

22. Hessler NA, Shirke AM, Malinow R: The probability of transmitter release at a mammalian central synapse. Nature 1993, 366(6455):569-572.

23. Rosenmund C, Clements JD, Westbrook GL: Nonuniform probability of glutamate release at a hippocampal synapse. Science 1993, 262(5134):754-757

24. Isaacson JS, Solis JM, Nicoll RA: Local and diffuse synaptic actions of GABA in the hippocampus. Neuron 1993, 10(2):165-175.

25. MCNaughton BL: Long-term synaptic enhancement and short-term potentiation in rat fascia dentata act through different mechanisms. J Physiol 1982, 324:249-262

26. Billups B, Graham BP, Wong AY, Forsythe ID: Unmasking group III metabotropic glutamate autoreceptor function at excitatory synapses in the rat CNS. J Physiol 2005, 565(Pt 3):885-896.
27. Dittman JS, Regehr WG: Calcium dependence and recovery kinetics of presynaptic depression at the climbing fiber to Purkinje cell synapse. J Neurosci 1998, 18(16):6147-6162

28. Junge HJ, Rhee JS, Jahn O, Varoqueaux F, Spiess J, Waxham MN, Rosenmund C, Brose N: Calmodulin and Munc13 form a Ca2+ sensor/ effector complex that controls short-term synaptic plasticity. Cell 2004, 118(3):389-401.

29. Sakaba T, Neher E: Calmodulin mediates rapid recruitment of fastreleasing synaptic vesicles at a calyx-type synapse. Neuron 2001 , 32(6):1119-1131.

30. Stevens CF, Wesseling JF: Activity-dependent modulation of the rate at which synaptic vesicles become available to undergo exocytosis. Neuron 1998, 21(2):415-424.

31. Wang LY, Kaczmarek LK: High-frequency firing helps replenish the readily releasable pool of synaptic vesicles. Nature 1998, 394(6691):384-388.

32. Allen NJ, Barres BA: Signaling between glia and neurons: focus on synaptic plasticity. Curr Opin Neurobiol 2005, 15(5):542-548

33. Santello $M$, Volterra $A$ : Synaptic modulation by astrocytes via Ca2 +-dependent glutamate release. Neuroscience 2009, 158(1):253-259.

34. Lisman J, Buzsaki G: A neural coding scheme formed by the combined function of gamma and theta oscillations. Schizophr Bull 2008, 34(5):974-980

35. Poncer JC, Malinow R: Postsynaptic conversion of silent synapses during LTP affects synaptic gain and transmission dynamics. Nat Neurosci 2001 4(10):989-996.

36. Hanse E, Gustafsson B: Quantal variability at glutamatergic synapses in area CA1 of the rat neonatal hippocampus. J Physiol 2001, 531(Pt 2):467-480

37. Hjelmstad GO, Isaac JT, Nicoll RA, Malenka RC: Lack of AMPA receptor desensitization during basal synaptic transmission in the hippocampal slice. J Neurophysiol 1999, 81(6):3096-3099.

38. Liu G, Choi S, Tsien RW: Variability of neurotransmitter concentration and nonsaturation of postsynaptic AMPA receptors at synapses in hippocampal cultures and slices. Neuron 1999, 22(2):395-409.

39. MCAllister AK, Stevens CF: Nonsaturation of AMPA and NMDA receptors at hippocampal synapses. Proc Natl Acad Sci USA 2000, 97(11):6173-6178.

40. Adesnik H, Nicoll RA: Conservation of glutamate receptor 2-containing AMPA receptors during long-term potentiation. J Neurosci 2007, 27(17):4598-4602.

41. Stubblefield EA, Benke TA: Distinct AMPA-type glutamatergic synapses in developing rat CA1 hippocampus. J Neurophysiol 2010, 104(4):1899-1912.

42. Matthias K, Kirchhoff F, Seifert G, Huttmann K, Matyash M, Kettenmann H, Steinhauser C: Segregated expression of AMPA-type glutamate receptors and glutamate transporters defines distinct astrocyte populations in the mouse hippocampus. J Neurosci 2003, 23(5):1750-1758.

doi:10.1186/1471-2202-12-87

Cite this article as: Andersson and Hanse: Astrocyte-mediated shortterm synaptic depression in the rat hippocampal CA1 area: two modes of decreasing release probability. BMC Neuroscience 2011 12:87.

\section{Submit your next manuscript to BioMed Central and take full advantage of:}

- Convenient online submission

- Thorough peer review

- No space constraints or color figure charges

- Immediate publication on acceptance

- Inclusion in PubMed, CAS, Scopus and Google Scholar

- Research which is freely available for redistribution 